\title{
Contribuição da citopatologia para o diagnóstico de carcinoma de células escamosas oral
}

\section{Contribution of cytopathology to the diagnosis of oral squamous cells carcinoma}

Karla Bianca F. C. Fontes'; Adrianna Milagres²; Maria Midori Miura Piragibe³; Licínio Esmeraldo da Silva4; Eliane Pedra Dias ${ }^{5}$

Unitermos
Carcinoma de células
escamosas oral
Citopatologia
Concordância diagnóstica

\section{resumo}

A incidência de câncer tem aumentado significativamente em todo o mundo, configurando-se como um dos mais importantes problemas de saúde pública. Como conseqüência o câncer de boca também tem aumentado, com $94 \%$ dos casos correspondendo ao carcinoma de células escamosas. O objetivo deste estudo foi atualizar o tema e documentar a experiência de cinco anos na utilização da citopatologia para avaliação de lesões orais suspeitas de malignidade. Realizou-se uma busca no arquivo do Serviço de Anatomia Patológica do Hospital Universitário Antônio Pedro da Universidade Federal Fluminense (HUAP/UFF), referente ao período de 2002 a 2007, de pacientes com lesões orais clinicamente suspeitas de carcinomas submetidos simultaneamente à biopsia incisional e a raspados da lesão para análise citopatológica. A amostra selecionada correspondeu a 50 casos, mas em 41 (82\%) pacientes, o diagnóstico histopatológico foi de carcinoma de células escamosas. Desses, a citopatologia foi conclusiva em 30 (73\%) e descreveu alterações suspeitas em 11 (27\%) pacientes. Os resultados revelaram que a citopatologia apresentou concordância diagnóstica de $74 \%$ com a histopatologia (padrão-ouro) nos diagnósticos positivos para carcinoma de células escamosas, e negativos para malignidade. Os resultados obtidos indicam a necessidade de padronização da técnica e da avaliação de grandes casuísticas para definição da acurácia, pois, na casuística avaliada, a citopatologia apresentou-se como um método fidedigno, que poderá ser utilizado como recurso de diagnóstico suficiente para o imediato encaminhamento dos pacientes para o tratamento de carcinoma de células escamosas oral.

\section{abstract}

Cancer is one of the most important problems in public health and its incidence has risen significantly worldwide. Accordingly, the incidence of oral cancer has also increased and $94 \%$ of the cases are caused by squamous cell carcinomas. The objective of the present study was to provide an update on the subject and report our five-year experience in the use of cytopathology analyzing suspected malignant oral lesions. We searched the slide archives of the anatomic pathology service of Hospital Universitário Antônio Pedro, Universidade Federal Fluminense (UFF), from 2002 to 2007. We selected patients with suspected carcinoma oral lesions who underwent incisional biopsy and were scraped for cytopathological analysis simultaneously. The sample consisted of 50 cases, of which 41 (82\%) were diagnosed with squamous cell carcinoma. Among these, cytopathology was conclusive in 30 cases (73\%) and suspected alterations were described in 11 (27\%). Results indicated that cytopathology presented diagnostic concordance with histopathology in positive diagnosis for squamous cell carcinoma and in negative for malignancy in $74 \%$ of the cases (gold standard). Our findings demonstrate that the standardization of techniques and the evaluation of a larger number of patients are required to define the accuracy of the method. In the studied sample cytopathology was a reliable method that may be used as a sufficient diagnostic tool to refer patients for immediate treatment of oral squamous cell carcinoma. key words

Oral squamous cell

carcinoma

Cytopathology

Diagnostic concordance

1. Mestranda do Programa de Pós-graduação em Patologia da Faculdade de Medicina da Universidade Federal Fluminense (UFF).

2. Doutoranda do Programa de Pós-graduação em Patologia da UFF.

3. Médica citopatologista do Instituto Nacional do Câncer (INCa).

4. Professor adjunto do Departamento de Estatística da UFF.

5. Professora titular do Departamento de Patologia da Faculdade de Medicina da UFF. 


\section{Introdução}

O número de casos de câncer tem aumentado de maneira significativa em todo o mundo, principalmente a partir do século passado, configurando-se atualmente em um dos mais importantes problemas de saúde pública mundia|( ${ }^{(34)}$. Conseqüentemente, a incidência de câncer de boca também está em ascensão, sendo o sexto tumor mais comum em todo mundo. Nos EUA, cerca de 40 mil casos são diagnosticados a cada ano ${ }^{(12)}$.

Segundo a estimativa de incidência de câncer de boca no Brasil para 2008, foram estimados 10.380 novos casos (quinta posição) entre os homens e 3.780 (sétima posição) entre as mulheres ${ }^{(17)}$.

De todos os tumores malignos que afetam a boca, cerca de $94 \%$ correspondem ao carcinoma de células escamosas oral (CCEO)(21). O CCEO é uma neoplasia epitelial maligna agressiva, que acomete principalmente o sexo masculino na faixa etária dos 50 aos 80 anos. Entretanto, as incidências de casos entre homens e mulheres estão se aproximando, provavelmente devido ao aumento da exposição a agentes carcinogênicos ${ }^{(21,22)}$. De etiologia desconhecida, vários fatores parecem estar envolvidos, tanto extrínsecos quanto intrínsecos. Os fatores extrínsecos incluem o fumo de tabaco, tabaco sem fumaça, masca de bétel, álcool, exposição aos fenóis, infecções por Candida e por vírus oncogênicos, bem como sífilis ${ }^{(21,33)}$. Como fatores intrínsecos enquadram-se os estados sistêmicos ou generalizados como desnutrição, anemia por deficiência de ferro, deficiência de vitamina A e suscetibilidade genética ${ }^{(21,33)}$.

A freqüência de CCEO é maior em pacientes com lesões epiteliais precursoras: leucoplasia, com potencial de transformação maligna de 1,3\% a 47\%; eritroplasia, com 91\% das lesões já apresentando displasia epitelial grave e a eritroleucoplasia ${ }^{(13,18,21,26,33)}$. De apresentação clínica variada, o CCEO pode exibir aspecto endofítico ou, mais freqüentemente, exofítico em que caracteristicamente apresenta-se com superfície irregular fungiforme, papilar ou verrucosa, e padrão de crescimento caracterizado por ulceração irregular, com borda elevada, vermelha ou branca, e firme ${ }^{(21)}$.

Há unanimidade na literatura científica em relação à língua ser a localização preferencial para o desenvolvimento de CCEO, seguida pelo assoalho da boca ${ }^{(4,21,25)}$. No entanto, não há consenso em relação à freqüência dos outros sítios anatômicos.

Segundo Neville et al.(21), as localizações intra-orais, em ordem decrescente de freqüência, após a língua e o assoalho da boca são: gengiva, mucosa jugal, mucosa labial e palato duro.

Portanto, considerando o melhor prognóstico dos CCEO diagnosticados e tratados nas fases iniciais e o alto percentual, mesmo nos países desenvolvidos, de pacientes que iniciam o tratamento nas fases mais avançadas, nas quais freqüentemente já são identificadas metástases para linfonodos regionais, o grande desafio é realizar o diagnóstico e iniciar o tratamento preferencialmente na fase intra-epitelial ou superficialmente invasora ${ }^{(15,32)}$.

A citopatologia é um método que se fundamenta na possibilidade de analisar as células coletadas das lesões e interpretar, à microscopia de campo claro, o esfregaço corado obtido a partir do material coletado. Em lesões orais, as células são obtidas pela raspagem na superfície da lesão suspeita, possibilitando a análise das características citopatológicas e a classificação da lesão(2). Essa técnica é bem aceita pelos pacientes, aspecto esse que pode ser bastante útil para o diagnóstico ${ }^{(15)}$. Entre as muitas vantagens, a citopatologia se destaca por ser rápida; de custo baixo; não-invasiva, pois o material é facilmente coletado; por não necessitar de anestesia infiltrativa, sendo o incômodo eliminado com a utilização de anestésico tópico; apresentar alta especificidade e sensibilidade, o que viabiliza a sua utilização como exame de rotina; minimizar a possibilidade de infecção e hemorragia em pacientes imunossuprimidos; permitir a aplicação de novas técnicas quantitativas, citomorfológicas, de imunocitoquímica e de biologia molecular, proporcionando maior precisão no diagnóstico $(7,8,11,15,19,23,30)$. Outro aspecto interessante é a identificação de células anormais utilizando programas especializados de computador, que pode agilizar a análise dos esfregaços, o diagnóstico do patologista e o tratamento ${ }^{(31,32)}$.

Entre as limitações do método, destaca-se a visualização apenas de células isoladas, impossibilitando a observação das alterações arquiteturais e a ocorrência de resultados falso-positivos e/ou falso-negativos. Esses têm sido justificados principalmente por erros na execução técnica; pela distribuição não-aleatória das células no esfregaço; por esfregaços muito espessos originando sobreposição celular significativa e pela grande quantidade de células sangüíneas e debris $(10,16)$.

Gindzienski et al.(9) afirmaram que a sobreposição de células nos esfregaços pode ser o resultado da aglutinação proporcionada pela presença de saliva sobre a lesão no momento da coleta de material. Do mesmo modo, Martins et al. ${ }^{(14)}$ demonstraram que a saliva é capaz de reduzir a quantidade de células coletadas e alterar a qualidade 
das lâminas, principalmente em relação à sobreposição de células, interferindo de forma negativa no diagnóstico citopatológico.

Portanto, levando em consideração a diversidade de aspectos clínicos dos CCEOs e os vários diagnósticos diferenciais, é indispensável uma definição diagnóstica para que o paciente possa ser submetido aos protocolos terapêuticos. Essa definição obrigatoriamente tem sido feita pela análise histopatológica de uma biópsia, habitualmente incisional( ${ }^{(8)}$. Contudo, a literatura relata que o método citopatológico aumentou a sua acurácia nas últimas décadas e tem sido considerado suficiente para definição diagnóstica em lesões da tireóide, mama, pulmão, mediastino, rim, bexiga, pâncreas e próstata ${ }^{(6)}$. No entanto, não há consenso na literatura a respeito da eficácia da utilização da citopatologia no diagnóstico do $\mathrm{CCEO}^{(1,8,15)}$. Logo, partindo desses pressupostos, o objetivo deste estudo foi atualizar o tema e documentar uma análise retrospectiva da utilização da citopatologia para avaliação de lesões orais suspeitas de malignidade.

\section{Material e métodos}

Realizou-se busca no arquivo do Serviço de Anatomia Patológica do Hospital Universitário Antônio Pedro (HUAP/ UFF) dos exames de citopatologia oral realizados entre 2002 e 2007, em pacientes com lesões orais suspeitas de malignidade submetidos simultaneamente à biópsia incisional e a raspados da lesão para análise histopatológica. Dos 56 casos identificados, foram selecionados 50 cujos diagnósticos histopatológicos realizados em biópsias incisionais corresponderam a carcinoma de células escamosas ou de afecção não-neoplásica.

Em todos os casos foram realizados procedimentos técnicos rotineiros para análises citopatológica e histopatológica, respectivamente, mediante fixação pelo álcool a $95^{\circ}$ e formol tamponado a $10 \%$, bem como coloração pelos métodos de Papanicolaou e hematoxilina-eosina (HE). Dos casos selecionados foram registrados: a cor da pele, o sexo, a idade e o local preciso da lesão.

Todas as lâminas foram reavaliadas simultaneamente por um observador em treinamento, um experiente em citopatologia geral e outro em citopatologia geral e oral, baseando-se nos seguintes critérios:

- celularidade (suficiente ou insuficiente para análise);

- tipo celular predominante e disposição;

- anisocitose;
- pleomorfismo celular;

- anaplasia;

- alterações nucleares (hipercromasia, anisocariose, cariomegalia, multinucleação, espessamento acentuado e/ou irregular da membrana nuclear, cromatina grosseira ou irregular, nucléolos evidentes, múltiplos ou macronucléolos, e mitoses atípicas);

- ceratinização atípica associada a alterações nucleares.

Os diagnósticos citopatológicos obtidos foram agrupados em:

- positivo para carcinoma de células escamosas;

- suspeito para carcinoma de células escamosas;

- negativo para malignidade;

- material inadequado para análise citopatológica.

Os diagnósticos histopatológicos estabelecidos como padrão-ouro foram baseados nos critérios estabelecidos pela Organização Mundial da Saúde (OMS)(35).

Os resultados obtidos foram comparados e a concordância diagnóstica entre a citopatologia e a histopatologia foi observada pela proporção de diagnósticos concordantes, acompanhada do intervalo de confiança (IC) de 95\%. Para os casos de CCEO, utilizou-se o teste binomial (50\%-50\%), ao nível de significância de 0,05 (5\%), para comparar a proporção de casos em que a citopatologia foi capaz de detectar a presença de CCEO com aqueles em que não foi capaz de decidir pela sua presença.

Estratégia semelhante foi adotada para os casos negativos para malignidade, quando se comparou a proporção de casos em que a citopatologia foi capaz de decidir pela negatividade para malignidade com a proporção daqueles casos em que não foi capaz de decidir pela negatividade.

\section{Resultados}

A amostra de 50 lesões (Tabela 1) foi proveniente de 50 pacientes, 32 (64\%) homens e 18 (36\%) mulheres, com idades variando de 29 a 93 anos. Em 41 (82\%) pacientes, o diagnóstico histopatológico foi de carcinoma de células escamosas oral (Figuras 1A/C). Desses, a citopatologia fez o diagnóstico de CCEO (Figura 1B) em 30 (73\%) e descreveu alterações suspeitas de CCEO (Figura 1D) em 11 (27\%). Dos nove casos que a histopatologia não revelou malignidade (Figura 1E), a citopatologia foi negativa para malignidade em sete (Figura 1F) e em dois o material foi inadequado para análise (Tabela 1). O CCEO ocorreu mais freqüentemente em homens (66\%) na faixa etária entre 40 e 


\section{Tabela 1 Distribuição dos diagnósticos histopatológicos e citopatológicos na amostra estudada}

\begin{tabular}{llccccc}
\hline & \multicolumn{5}{c}{ Resultado citopatológico } \\
& $\begin{array}{c}\text { Positivo para } \\
\text { CCEO }\end{array}$ & $\begin{array}{c}\text { Suspeito para } \\
\text { CCEO }\end{array}$ & $\begin{array}{c}\text { Negativo para } \\
\text { malignidade }\end{array}$ & $\begin{array}{c}\text { Material } \\
\text { inadequado }\end{array}$ & Total \\
$\begin{array}{l}\text { Resultado } \\
\text { histopatológico }\end{array}$ & $\begin{array}{l}\text { CCEO } \\
\text { Negativo para } \\
\text { malignidade }\end{array}$ & -30 & 11 & - & - & 41 \\
Total & 30 & 11 & 7 & 2 & 9 \\
\hline CCEO carcinoma de céluas escamosas oral & & & - & 7 & 50 \\
\hline
\end{tabular}

CCEO: carcinoma de células escamosas oral.

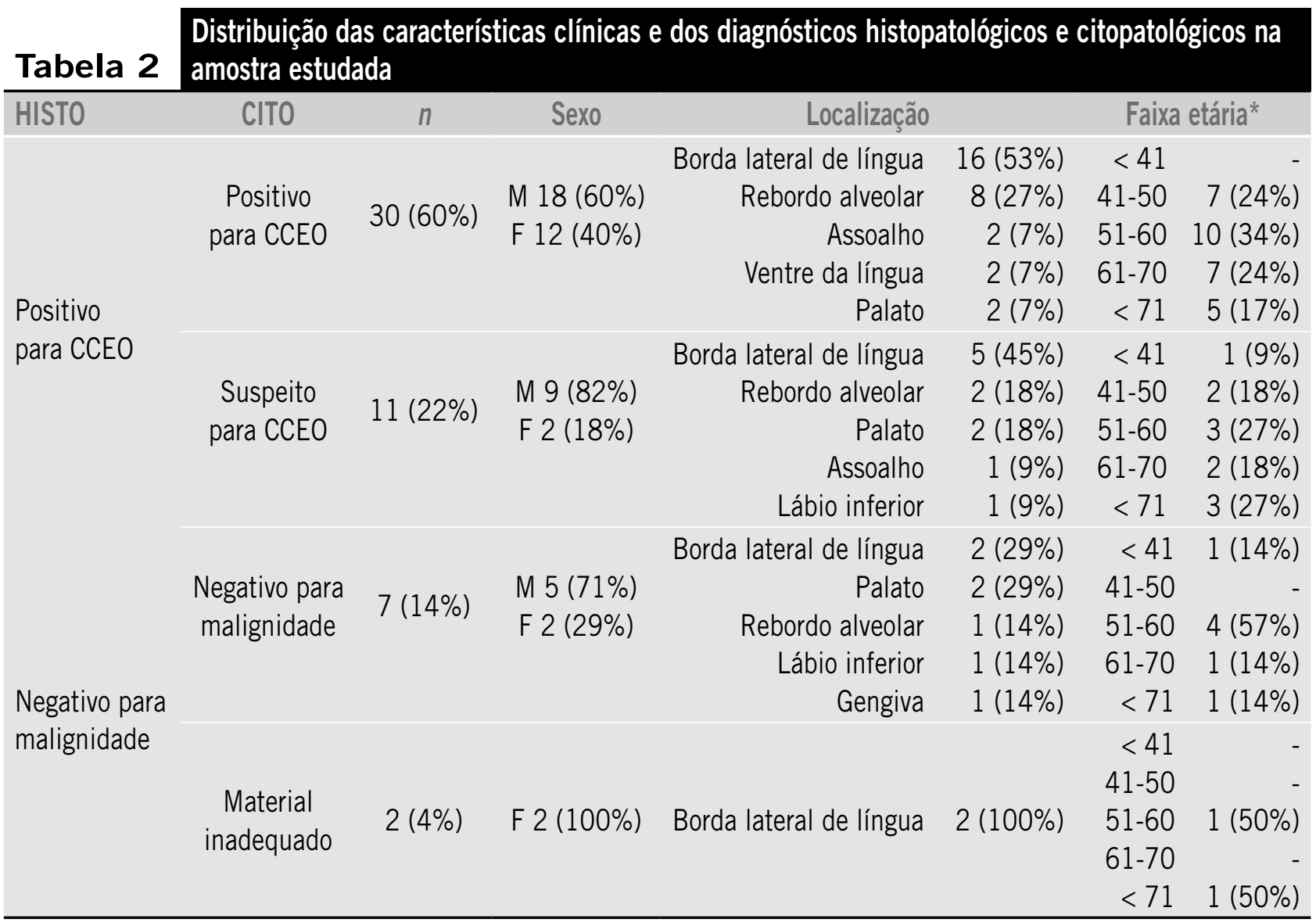

* Ausência de informação em um paciente.

HISTO: diagnóstico histopatológico realizado em biópsias incisionais; $n$ : número de casos; CITO: diagnóstico citopatológico realizado em esfregaços realizados nas lesões biopsiadas; M: sexo masculino; F: sexo feminino; CCEO: carcinoma de células escamosas oral

70 anos (76\%). Dos 41 pacientes com diagnóstico de CCEO, 10 (24\%) tinham menos de 50 anos. Preferencialmente, os CCEOs localizaram-se em borda lateral da língua (51\%), com rebordo alveolar apresentando percentual de $24 \%$.

A concordância diagnóstica entre os resultados citopatológicos e histopatológicos foi de 74\% - IC: 61,85\%-86,15\%. Nos 41 casos de CCEOs definidos pela histopatologia, a citopatologia apresentou diagnóstico concordante em $73 \%$ dos casos $(n=30)$ e em $27 \%(n=11)$ dos casos não foi possível decidir pela positividade. Essas duas proporções foram diferentes com significância estatística, segundo o resultado do teste binomial (50\%-50\%) $(p=0,005)$, o que permitiu concluir que a citopatologia apresenta capacidade diagnóstica maior do que a impossibilidade de decidir pelos casos de CCEO.

Nos nove casos negativos para malignidade definidos pela histopatologia, a citopatologia apresentou concordância diagnóstica em $77,8 \%$ dos casos $(n=7)$ e no 




Figura 1A - Histopatologia: ninhos de células epiteliais escamosas atípicas entremeadas por conjuntivo denso, exibindo aumento da relação núcleo-citoplasma, graus variados de pleomorfismo nuclear e mitoses atípicas, correspondentes a carcinoma de células escamosas moderadamente diferenciado. Coloração por hematoxilina-eosina (HE), aumento inicial de 200x. B - Citopatologia: esfregaço correspondente ao raspado da lesão apresentando grupamento de células escamosas atípicas e exibindo aumento da relação núcleocitoplasma, citoplasma denso, hipercromasia nuclear, cariomegalia, anisocariose, membrana nuclear irregular e nucléolo evidente, correspondentes a carcinoma de células escamosas. Coloração por Papanicolaou, aumento inicial de 200x. C - Histopatologia: células epiteliais escamosas atípicas entremeadas por conjuntivo denso e exibindo aumento da relação núcleo-citoplasma, centralizadas por pérolas de queratina, correspondente a carcinoma de células escamosas. Coloração por HE, aumento inicial de 100x. D - Citopatologia: esfregaço correspondente ao raspado da lesão apresentando grupamento de células epiteliais com alteração da relação núcleo-citoplasma, citoplasma denso e cariomegalia. Aspectos sugestivos de carcinoma de células escamosas. Coloração por Papanicolaou, aumento inicial de 200x. E - Histopatologia: fragmento de mucosa apresentando epitélio escamoso estratificado paraqueratinizado, acantose, espongiose e leve exocitose. No conjuntivo subepitelial observa-se leve infiltrado inflamatório mononuclear, configurando aspectos de mucosite inespecífica. Coloração por HE, aumento inicial de 200x. F - Citopatologia: esfregaço correspondente ao raspado da lesão apresentando células escamosas típicas, sem aspectos citopatológicos de malignidade. Coloração por Papanicolaou, aumento inicial de 400x 
restante, $22,2 \%(n=2)$, não foi capaz de decidir pela negatividade. Essas duas proporções apresentaram-se estatisticamente equivalentes segundo o resultado do teste binomial (50\%-50\%) $(p=0,180)$, o que permitiu concluir que a citopatologia na amostra avaliada não apresenta capacidade diagnóstica suficiente para a detecção da negatividade.

\section{Discussão}

Nossos resultados confirmaram a maior prevalência de CCEO no sexo masculino (66\%), na faixa etária entre 40 e 70 anos (76\%), ocorrendo preferencialmente em borda lateral de língua (51\%). O rebordo alveolar foi a segunda localização mais freqüente (24\%), mas como não é uma localização relatada com freqüência pela literatura, pode tratar-se de casuísmo e/ou particularidade regional.

O câncer de boca em estágio avançado não costuma ser uma dificuldade diagnóstica na prática clínica. $\mathrm{O}$ grande desafio é considerar a possibilidade de a lesão ser maligna, a despeito da sua aparência inofensiva, especialmente porque o diagnóstico inicial do câncer de boca raramente é realizado pela maioria dos profissionais que examinam a boca. O prognóstico dos CCEOs diagnosticados e tratados em sua fase inicial é muito satisfatório ${ }^{(15)}$. Nesses, devido aos tratamentos menos complexos e menos agressivos, $80 \%$ dos pacientes alcançam maior taxa de sobrevida de cinco anos, com boa qualidade de vida após o tratamento(15).

A especial facilidade de acesso às lesões orais tem sido um ponto crítico nas discussões sobre diagnóstico precoce do câncer oral. É inquestionável a dificuldade mundial para reduzir o número de CCEOs diagnosticados em fase tardia, sendo necessária uma criteriosa análise processual. Empiricamente o maior obstáculo parece residir na identificação de lesões epiteliais precursoras, o que implica na necessidade de maior investimento no treinamento dos profissionais de saúde, particularmente cirurgiões-dentistas e médicos, para identificar essas lesões e proceder ao encaminhamento adequado.A rapidez da definição diagnóstica é uma condição importante para o tratamento efetivo do câncer, aspecto que pode ser positivamente alterado pela utilização da citopatologia ${ }^{(5,15)}$. Apesar de ser pouco utilizada entre os profissionais das áreas odontológica e médica, possivelmente por não ter sido reconhecida como ferramenta diagnóstica eficiente, a citopatologia deve ser, em nossa opinião, considerada como possível método definidor de diagnóstico.
Com o intuito de minimizar os fatores que influenciam a qualidade da amostra, Ogden et al. ${ }^{(24)}$ indicaram que, antes de iniciar a coleta da amostra, deve-se considerar a presença de ceratina no tecido, a quantidade de saliva no local, a visibilidade da área de eleição, a experiência do profissional que realiza a coleta da amostra e o instrumento empregado para a coleta. Também verificaram que a escova endocervical cytobrush é significativamente mais eficiente do que a espátula de madeira, em termos de quantidade de células obtidas, visualização do campo de célula e dispersão celular.

A visualização apenas de células isoladas, sem as alterações arquiteturais, tem sido considerada uma limitação do método citopatológico por aumentar a possibilidade de falso-positivos e falso-negativos ${ }^{(10,16)}$. A reavaliação dos esfregaços selecionados para este estudo, realizada por observadores em diferentes níveis de experiência, possibilitou a percepção de que essas limitações podem ser superadas, pois a presença de sobreposição celular, de células estromais e sangüíneas numerosas não impede a realização do diagnóstico correto. Soma-se o fato de que, para o profissional experiente, a distribuição das células no esfregaço, os arranjos peculiares, a associação cito-histopatológica, o conhecimento das várias doenças e a correlação clínica são dados utilizados para aumentar a acurácia diagnóstica.

A sensibilidade, a especificidade, os valores preditivos (positivo e negativo), a acurácia e os resultados falso-positivos e falso-negativos têm sido documentados em diversos estudos que comparam os métodos de diagnósticos citopatológicos e histopatológicos de pacientes com lesões suspeitas de carcinoma oral.

Embora Campagnoli e Lima( ${ }^{(3)}$ não tenham obtido bons resultados com a citopatologia convencional, Ramaesh et al. ${ }^{(28)}$, Kaugars et al. ${ }^{(11)}$, Roco Perez et al. ${ }^{(30)}$, Navone et al. ${ }^{(20)}$ relataram acurácia diagnóstica, respectivamente, de $95 \%$, $90 \%, 96 \%$ e $89,6 \%$, concluindo que o exame citopatológico é extremamente confiável e que pode ser empregado para o diagnóstico de neoplasias orais. Apesar de a casuística ainda ser limitada, este estudo sugere que a citopatologia realizada por citopatologistas experientes é um método diagnóstico confiável, pois revelou $74 \%$ de concordância diagnóstica entre todos os resultados citopatológicos e histopatológicos. Esses resultados são indicadores de que as alterações celulares são suficientes, na maioria das vezes, para a realização de um diagnóstico conclusivo e que a arquitetura tecidual, ainda que importante, pode não ser um elemento indispensável para o diagnóstico de malignidade e para a classificação do tipo tumoral. 
Rick e Slater ${ }^{(29)}$ observaram resultados falso-positivos na técnica de citopatologia, apresentando apenas 3\% de especificidade. Entretanto, em estudo realizado por Potter et al. (27), apenas quatro resultados falso-negativos (3,5\%) foram encontrados em 115 CCEOs. Não identificamos na amostra avaliada resultados falso-positivos ou falso-negativos.

Moraes et al. ${ }^{(19)}$ relataram que a citopatologia apresenta sensibilidade, especificidade, valores preditivos (positivo e negativo) e precisão de $100 \%$, quando considerada a capacidade de descartar malignidade. Comprovamos que a citopatologia confirmou o resultado histopatológico de ausência de malignidade em todos os esfregaços em que o material estava adequado para avaliação citopatológica.

De acordo com Czerninski e Markitziu( ${ }^{(5)}$, somente especialistas experientes, que saibam diferenciar lesão suspeita de malignidade de tumor benigno local ou de desordem sistêmica, poderiam utilizar a técnica de citopatologia de maneira efetiva. Acredita-se que a presença de um citopatologista experiente é fundamental para evitar erros de diagnóstico.

A literatura é unânime em considerar a citopatologia um método simples, de fácil execução, rápido, com baixo custo, não-invasivo, relativamente indolor, inócuo e bem aceito pelos pacientes $(7,8,11,15,19,23,30)$. Possivelmente a discrepância observada nos valores de alguns estudos é proveniente da não-padronização da técnica, da utilização de diferentes testes de análises estatísticas, de números de amostras não representativos, de diferentes instrumentos empregados para a coleta do material, da eficiência do profissional que realiza a coleta da amostra e, principalmente, da experiência ou inexperiência do profissional que realizou o citodiagnóstico.

\section{Comentários finais}

Os resultados deste estudo indicam que a citopatologia pode ser utilizada rotineiramente como método diagnóstico de carcinoma de células escamosas oral, já que apresenta elevada concordância diagnóstica com o exame histopatológico (padrão-ouro) nos diagnósticos de positividade para carcinoma de células escamosas, e negatividade para malignidade. Acreditamos que a avaliação de maiores casuísticas e a padronização da técnica poderão definir a acurácia do método, aspecto ainda controverso na literatura mundial. Essa definição poderá beneficiar significativamente os pacientes que tiverem seus diagnósticos definidos pela citopatologia, já que poderão ser imediatamente encaminhados para o tratamento.

\section{Referências}

1. ACHA, A. et al. Applications of the oral scraped (exfoliative) cytology in oral cancer and precancer. Med Oral Patol Oral Cir Bucal, v. 10, n. 2, p. 95-102, 2005.

2. BIRMAN, E. G.; SUGAYA, N. N. Citologia no diagnóstico do câncer bucal. In: KOWALSKI, L. P. et al. Prevenção, diagnóstico e tratamento do câncer bucal. Hospital do Câncer e Associação Paulista de Cirurgiões Dentistas. São Paulo: Frôntis Editorial, 1999.

3. CAMPAGNOLI, E. B; LIMA, A. A. S. Comparação entre a citologia em base líquida e a citologia convencional no diagnóstico de carcinomas bucais. Paraná, 2003. Dissertação de Mestrado em Odontologia (área de concentração Estomatologia), Faculdade de Odontologia da Pontifícia Universidade Católica do Paraná.

4. CARVALHO, M. B. et al. Clinical and epidemiological characteristics of oral squamous cell carcinoma in women. Rev Assoc Med Bras, v. 47, n. 3, p. 208-14, 2001.

5. CZERNINSKI, R.; MARKITZIU, A. Only fully trained oral medicine clinicians should use cytobrush. Oral Surg Oral Med Oral Pathol Oral Radiol Endod, v. 94, n. 6, p. 655-7, 2002.

6. DEMAY, R. M. The art and science of cytopathology. Hong Kong: ASCP Press, 1996. V. 1-2.
7. DINIZ FREITAS, M. et al. Applications of exfoliative cytology in the diagnosis of oral cancer. Med Oral Patol Oral Cir Bucal, v. 9, n. 4, p. 355-61, 2004.

8. EPSTEIN, J. B. et al. Advances in the diagnosis of oral premalignant and malignant lesions. J Can Dent Assoc, v. 68, p. 617-21, 2002.

9. GINDZIENSKI, A. et al. The role of mucus and its components in protection and repair within the alimentary tract mucosa: Polish experience. J Physiol Pharmacol, v. 54, n. S3, p. 127-44, 2003.

10. KAHN, M. A. Oral exfoliative cytology procedures: conventional, brush biopsy and Thin Prep. J Tenn Dental Assoc, v. 81, n. 1, p. 17-20, 2001.

11. KAUGARS, G. E. et al. The use of exfoliative cytology for the early diagnosis of oral cancers: is there a role for it in education and private practice? J Cancer Educ, v. 13, n. 2, p. 85-9, 1998.

12. LINGEN, M. W.; KUMAR, V. Cabeça e pescoço. In: KUMAR, V.; ABBAS, A.; FAUSTO, N. Patologia: bases patológicas das doenças. 7. ed. Rio de Janeiro: Elsevier, 2005. Cap. 16; p. 820.

13. LUMERMAN, H. et al. Oral epithelial dysplasia and the development of invasive squamous cell carcinoma. Oral 
Surg, Oral Med, Oral Pathol, Oral Radio, and Endod, v. 79, n. 3, p. 321-9, 1995.

14. MARTINS, D. D. M. et al. Interferência da saliva na qualidade das lâminas de lesões bucais obtidas pela citologia esfoliativa em meio líquido. Rev Bras de Patol Oral, v. 4, n. 3, p. 136-42, 2005.

15. MEHROTRA, R. etal. Application of cytology and molecular biology in diagnosing premalignant or malignant oral lesions. Molecular Cancer, v. 5, n. 11, 2006.

16. MERLIN, J. C. Citologia cérvico-vaginal: estudo dos métodos colpocitológicos convencional e de monocamada ou base líquida, baseado na celularidade retida em instrumentos de coleta. Paraná, 2002. Dissertação (Mestrado em Biologia Celular) - Faculdade de Biologia, Universidade Federal do Paraná.

17. MINISTÉRIO DA SAÚDE; INSTITUTO NACIONAL DO CÂNCER. Estimativa para 2008 do número de casos novos e respectivas taxas brutas para neoplasias, 2007. Disponível em: http:www.inca.gov.br/. Acesso em: 12 dez. 2007.

18. MISHRA, M. et al. Epidemiological and clinicopathological study of oral leukoplakia. Indian J Dermatol Venerol Leprol, v. 71, n. 3, p. 161-5, 2005.

19. MORAES, M. et al. Atlas virtual de citologia esfoliativa em lesões de boca. Pesquisa Odontol Bras, Brasília, 2005. Disponível em: http:www.unb.br/fs/citovirtual/artigo1. pdf. Acesso em: 10 jun. 2006.

20. NAVONE, R. et al. Usefulness of oral exfoliative cytology for the diagnosis of oral squamous dysplasia and carcinoma. Minerva Stomatol, v. 53, n. 3, p. 77-86, 2004.

21. NEVILLE, B. W. et al. Patología Epitelial. In: _. Patologia oral \& maxilofacial. 2. ed. Rio de Janeiro: GuanabaraKoogan, 2004. Cap. 10; p. 325-54.

22. NEVILLE, B. W.; DAY, T. A. Oral cancer and precancerous lesions. CA J Clinic, v. 52, n. 4, p. 195-215, 2002.

23. NICHOLS, M. L. et al. Interobserver variability in the interpretation of brush cytologic studies from head and neck lesions. Arch Otolaryngol Head Neck Sur, v. 117, n. 12, p. 1350-5, 1991.

24. OGDEN, G. R.; COWPE, J. G.; GREEN, M. Cytobrush and wooden spatula for oral exfoliative cytology: a comparison. Acta Cytol, v. 36, n. 5, p. 706-10, 1992.
25. OLAS, O. L. A. S. G. et al. Oral squamous cell carcinoma: a retrospective study of 740 cases in a Brazilian population. Braz Dent J, v. 12, n. 1, p. 57-61, 2001.

26. PETTI, S. Pooled estimate of world leukoplakia prevalence a systematic review. Oral Oncol, v. 39, n. 8, p. 77080, 2003.

27. POTTER, T. J.; SUMMERLIN, D. J.; CAMPBELL, J. $H$. Oral malignancies associated with negative transepithelial brush biopsy. J Oral Maxillofac Surg, v. 61, p. 674-7, 2003.

28. RAMAESH, C. et al. Exfoliative cytology in secreening for malignant and premalignant lesions in the buccal mucosa. Ceylon Medical Journal, v. 43, n. 4, p. 206-9, 1998

29. RICK, G. M.; SLATER, L. Oral brush biopsy: the problem of false positives. Oral Surg Oral Med Oral Pathol Oral Radiol Endod, v. 96, p. 252, 2003.

30. ROCO PEREZ, O. G.; ARREDONDO LOPEZ, M.; ALVAREZ NAVARRO, M. D. C. Citología exfoliativa en el diagnóstico precoz de lesiones oncológicas bucales. Rev Cubana Estomatol, v. 39, n. 2, p. 89-100, 2002.

31. SCHEIFELE, C. et al. The sensitivity and specificity of the OralCDx technique: evaluation of 103 cases. Oral Oncol, v. 40 , n. 8, p. 824-8, 2004.

32. SCIUBBA, J. J. Improving detection of precancerous and cancerous oral lesions: computer-assisted analysis of the oral brush biopsy. U.S. Collaborative OralCDx Study Group. Am Dent Assoc, v. 130, n. 10, p. $1445-$ 57, 1999.

33. SILVERMAN, S.; EVERSOLE, L. R. Lesões pré-malignas e carcinoma de células escamosas bucais. In: SILVERMAN, S.; EVERSOLE, L. R.; TRUELOVE, E. L. Fundamentos de medicina oral. 2. ed. Rio de Janeiro: Guanabara Koogan, 2004. Cap. 20; p. 185-204.

34. WORLD HEATH ORGANIZATION (WHO). Policies and managerial guidelines for national cancer control programs. Rev Panam Salud Publ, v. 12, n. 5, p. 36670, 2002.

35. WORLD HEATH ORGANIZATION (OMS). World Heath Organization classification of tumours: pathology \& genetics head and neck tumours. Lyon: IARC Press, 2005; p. 163-207. 